\title{
Research Article \\ Best Approximations Theorem for a Couple in Cone Banach Space
}

\author{
Erdal Karapınar ${ }^{1}$ and Duran Türkoğlu \\ ${ }^{1}$ Department of Mathematics, Atılım University, 06836 Incek, Ankara, Turkey \\ ${ }^{2}$ Department of Mathematics, Gazi University, 06500 Teknikokullar, Ankara, Turkey \\ Correspondence should be addressed to Erdal Karapınar, erdalkarapinar@yahoo.com
}

Received 23 March 2010; Accepted 8 June 2010

Academic Editor: A. T. M. Lau

Copyright (C) 2010 E. Karapınar and D. Türkoğlu. This is an open access article distributed under the Creative Commons Attribution License, which permits unrestricted use, distribution, and reproduction in any medium, provided the original work is properly cited.

The notion of coupled fixed point is introduced by Bhaskar and Lakshmikantham, (2006). In this manuscript, some result of Mitrović, (2010) extended to the class of cone Banach spaces.

\section{Introduction and Preliminaries}

Banach, valued metric space was considered by Rzepecki [1], Lin [2], and lately by Huang and Zhang [3]. Basically, for nonempty set $X$, the definition of metric $d: X \times X \rightarrow \mathbb{R}^{+}=[0, \infty)$ is replaced by a new metric, namely, by an ordered Banach space $E: d: X \times X \rightarrow E$. Such metric spaces are called cone metric spaces (in short CMSs). In 1980, by using this idea Rzepecki [1] generalized the fixed point theorems of Maia type. Seven years later, Lin [2] extends some results of Khan and Imdad [4] by considering this new metric space construction. In 2007, Huang and Zhang [3] discussed some properties of convergence of sequences and proved the fixed point theorems of contractive mapping for cone metric spaces: any mapping $T$ of a complete cone metric space $X$ into itself that satisfies, for some $0 \leq k<1$, the inequality

$$
d(T x, T y) \leq k d(x, y)
$$

for all $x, y \in X$, has a unique fixed point. Recently, many results on fixed point theorems have been extended to cone metric spaces (see, e.g., [3, 5-11]). In [3], the authors extends to cone metric spaces over regular cones. In this manuscript, some results of some result of Mitrović in [12] are extended to the class of cone metric spaces. 
Throughout this paper $E$ stands for real Banach space. Let $P:=P_{E}$ always be closed subset of E.P is called cone if the following conditions are satisfied:

(C1) $P \neq \emptyset$,

(C2) $a x+b y \in P$ for all $x, y \in P$ and nonnegative real numbers $a, b$,

(C3) $P \cap(-P)=\{0\}$ and $P \neq\{0\}$.

For a given cone $P$, one can define a partial ordering (denoted by $\leq$ or $\leq_{P}$ ) with respect to $P$ by $x \leq y$ if and only if $y-x \in P$. The notation $x<y$ indicates that $x \leq y$ and $x \neq y$ while $x \ll y$ will show $y-x \in \operatorname{int} P$, where int $P$ denotes the interior of $P$. It can be easily shown that int $P+\operatorname{int} P \subset$ int $P$ and $\lambda($ int $P) \subset$ int $P$ where $0<\lambda \in \mathbb{R}$. Throughout this manuscript int $P \neq \emptyset$.

The cone $P$ is called

(N) normal if there is a number $K \geq 1$ such that for all $x, y \in E$,

$$
0 \leq x \leq y \Longrightarrow\|x\| \leq K\|y\|
$$

(R) regular if every increasing sequence which is bounded from above is convergent. That is, if $\left\{x_{n}\right\}_{n \geq 1}$ is a sequence such that $x_{1} \leq x_{2} \leq \cdots \leq y$ for some $y \in E$, then there is $x \in E$ such that $\lim _{n \rightarrow \infty}\left\|x_{n}-x\right\|=0$.

In $(N)$, the least positive integer $K$ satisfying (1.2) is called the normal constant of $(P)$. Note that, in $[3,5]$, normal constant $K$ is considered a positive real number, $(K>0)$, although it is proved that there is no normal cone for $K<1$ in (see e.g., Lemma 2.1, [5]).

Lemma 1.1 (see e.g., [13]). One has the following.

(i) Every regular cone is normal.

(ii) For each $k>1$, there is a normal cone with normal constant $K>k$.

(iii) The cone $P$ is regular if every decreasing sequence which is bounded from below is convergent.

Definition 1.2 (see [14]). $P$ is called minihedral cone if $\sup \{x, y\}$ exists for all $x, y \in E$; and strongly minihedral if every subset of $E$ which is bounded from above has a supremum.

Example 1.3. Let $E=C[0,1]$ with the supremum norm and $P=\{f \in E: f \geq 0\}$. Since the sequence $x^{n}$ is monotonically decreasing, but not uniformly convergent to 0 , thus, $P$ is not strongly minihedral.

Definition 1.4. Let $X$ be nonempty set. Suppose that the mapping $d: X \times X \rightarrow E$ satisfies the following:

(M1) $0 \leq d(x, y)$ for all $x, y \in X$,

(M2) $d(x, y)=0$ if and only if $x=y$,

(M3) $d(x, y) \leq d(x, z)+d(z, y)$, for all $x, y \in X$.

(M4) $d(x, y)=d(y, x)$ for all $x, y \in X$.

Then $d$ is called cone metric on $X$, and the pair $(X, d)$ is called a cone metric space (CMS). 
Example 1.5. Let $E=\mathbb{R}^{3}$ and $P=\{(x, y, z) \in E: x, y, z \geq 0\}$ and $X=\mathbb{R}$. Define $d: X \times X \rightarrow E$ by $d(x, \tilde{x})=(\alpha|x-\tilde{x}|, \beta|x-\tilde{x}|, \gamma|x-\tilde{x}|)$, where $\alpha, \beta, \gamma$ are positive constants. Then $(X, d)$ is a CMS. Note that the cone $P$ is normal with the normal constant $K=1$.

It is quite natural to consider Cone Normed Spaces (CNSs).

Definition 1.6 (see e.g., $[9,15,16]$ ). Let $X$ be a vector space over $\mathbb{R}$. Suppose that the mapping $\|\cdot\|_{P}: X \rightarrow E$ satisfies the following:

(N1) $\|x\|_{P}>0$ for all $x \in X$,

(N2) $\|x\|_{P}=0$ if and only if $x=0$,

(N3) $\|x+y\|_{P} \leq\|x\|_{P}+\|y\|_{P}$, for all $x, y \in X$.

$(N 4)\|k x\|_{P}=|k|\|x\|_{P}$ for all $k \in \mathbb{R}$.

Then $\|\cdot\|_{P}$ is called cone norm on $X$, and the pair $\left(X,\|\cdot\|_{P}\right)$ is called a cone normed space (CNS).

Note that each CNS is CMS. Indeed, $d(x, y)=\|x-y\|_{P}$.

Definition 1.7. Let $\left(X,\|\cdot\|_{P}\right)$ be a CNS, $x \in X$, and $\left\{x_{n}\right\}_{n \geq 1}$ a sequence in $X$. Then one has the following.

(i) $\left\{x_{n}\right\}_{n \geq 1}$ converges to $x$ whenever for every $c \in E$ with $0 \ll c$ there is a natural number $N$, such that $\left\|x_{n}-x\right\|_{P} \ll c$ for all $n \geq N$. It is denoted by $\lim _{n \rightarrow \infty} x_{n}=x$ or $x_{n} \rightarrow x$.

(ii) $\left\{x_{n}\right\}_{n \geq 1}$ is a Cauchy sequence whenever for every $c \in E$ with $0 \ll c$ there is a natural number $N$, such that $\left\|x_{n}-x\right\|_{P} \ll c$ for all $n, m \geq N$.

(iii) $\left(X,\|\cdot\|_{P}\right)$ is a complete cone normed space if every Cauchy sequence is convergent.

Complete cone-normed spaces will be called cone Banach spaces.

Lemma 1.8. Let $\left(X,\|\cdot\|_{P}\right)$ be a $C N S$, let $P$ be a normal cone with normal constant $K$, and let $\left\{x_{n}\right\}$ be a sequence in $X$. Then, one has the following:

(i) the sequence $\left\{x_{n}\right\}$ converges to $x$ if and only if $\left\|x_{n}-x\right\|_{P} \rightarrow 0$, as $n \rightarrow \infty$,

(ii) the sequence $\left\{x_{n}\right\}$ is Cauchy if and only if $\left\|x_{n}-x_{m}\right\|_{P} \rightarrow 0$ as $n, m \rightarrow \infty$,

(iii) the sequence $\left\{x_{n}\right\}$ converges to $x$ and the sequence $\left\{y_{n}\right\}$ converges to $y$ and then $\left\|x_{n}-y_{n}\right\|_{P} \rightarrow\|x-y\|_{P}$.

The proof is direct by applying Lemmas 1, 4, and 5 in [3] to the cone metric space $(X, d)$, where $d(x, y)=\|x-y\|_{P}$, for all $x, y \in X$.

Lemma 1.9 (see, e.g., $[6,7])$. Let $\left(X,\|\cdot\|_{P}\right)$ be a CNS over a cone $P$ in E. Then $(1) \operatorname{Int}(P)+\operatorname{Int}(P) \subseteq$ $\operatorname{Int}(P)$ and $\lambda \operatorname{Int}(P) \subseteq \operatorname{Int}(P), \lambda>0$. (2) If $c \gg 0$, then there exists $\delta>0$ such that $\|b\|<\delta$ implies $b \ll c$. (3) For any given $c \gg 0$ and $c_{0} \gg 0$ there exists $n_{0} \in \mathbb{N}$ such that $\left(c_{0} / n_{0}\right) \ll c$. (4) If $a_{n}, b_{n}$ are sequences in E such that $a_{n} \rightarrow a, b_{n} \rightarrow b$ and $a_{n} \leq b_{n}$, for all $n$, then $a \leq b$. 
Definition 1.10. Let $(X, d)$ be a CNS and let $I=[0,1]$ be the closed unit interval. A continuous mapping $W: X \times X \times I: \rightarrow X$ is said to be a convex structure on $X$ if for all $x, y \in X$ and $t \in I$

$$
\|u-W(x, y, t)\|_{P} \leq t\|u-x\|_{P}+(1-t)\|u-y\|_{P}
$$

holds for all $u \in X$. A CNS $(X, d)$ together with a convex structure is said to be convex CNS. A subset $Y \subset X$ is convex, if $W(x, y, t) \in Y$ holds for all $x, y \in X$ and $t \in I$.

Definition 1.11. Let $X$ be a CNS, and $K$ and $C$ the nonempty convex subsets of $X$. A mapping $g: K \rightarrow X$ is said to be almost quasiconvex with respect to $C$ if

$$
\|g(t x+(1-t) y)-z\| \leq c_{g}([t x+(1-t) y], z)
$$

where $c_{g}([t x+(1-t) y], z) \in\left\{\|g(x)-z\|_{P},\|g(y)-z\|_{P}\right\}$ for all $x, y \in K, z \in C$, and $0<t<1$.

\section{Couple Fixed Theorems on Cone Metric Spaces}

Let $(X, d)$ be a CMS and $X^{2}:=X \times X$. Then the mapping $\rho:=X^{2} \times X^{2}: \rightarrow E$ such that $\rho\left(\left(x_{1}, y_{1}\right),\left(x_{2}, y_{2}\right)\right):=d\left(x_{1}, x_{2}\right)+d\left(y_{1}, y_{2}\right)$ forms a cone metric on $X^{2}$. A sequence $\left(\left\{x_{n}\right\},\left\{y_{n}\right\}\right) \in X^{2}$ is said to be a double sequence of $X$. A sequence $\left(\left\{x_{n}\right\},\left\{y_{n}\right\}\right) \in X^{2}$ is convergent to $(x, y) \in X^{2}$ if, for every $c \in \operatorname{int}(P)$, there exists a natural number $M>0$ such that $\rho\left(\left(x_{n}, y_{n}\right),(x, y)\right) \ll c$ for all $n>M$.

Lemma 2.1. Let $z_{n}=\left(x_{n}, y_{n}\right) \in X^{2}$ and $z=(x, y) \in X^{2}$. Then, $z_{n} \rightarrow z$ if and only if $x_{n} \rightarrow x$ and $y_{n} \rightarrow y$.

Proof. Suppose $z_{n} \rightarrow z$. Thus, for any $c \in \operatorname{int}(P)$, there exist $M>0$ such that $\rho\left(\left(x_{n}, y_{n}\right),(x, y)\right)=d\left(x_{n}, x\right)+d\left(y_{n}, y\right) \ll c$ for all $n>M$. Hence, $d\left(x_{n}, x\right) \ll c$ and $d\left(y_{n}, y\right) \ll c$ for all $n>M$, that is, $x_{n} \rightarrow x$ and $y_{n} \rightarrow y$.

Conversely, assume $x_{n} \rightarrow x$ and $y_{n} \rightarrow y$. Thus, for any $c \in \operatorname{int}(P)$, there exist $M_{0}, M_{1}>0$ such that $d\left(x_{n}, x\right) \ll c / 2$ for all $n>M_{0}$, and also $d\left(y_{n}, y\right) \ll c / 2$ for all $n>M_{1}$. Hence, $\rho\left(\left(x_{n}, y_{n}\right),(x, y)\right)=d\left(x_{n}, x\right)+d\left(y_{n}, y\right) \ll c$ for all $n>M$, where $M:=\max \left\{M_{0}, M_{1}\right\}$.

Definition 2.2. Let $(X, d)$ be a CMS. A function $f: X \rightarrow X$ is said to be sequentially continuous if $d\left(x_{n}, x\right) \rightarrow 0$ implies that $d\left(f\left(x_{n}\right), f(x)\right) \rightarrow 0$. Analogously, a function $F: X \times X: \rightarrow X$ is sequentially continuous if $\rho\left(\left(x_{n}, y_{n}\right),(x, y)\right) \rightarrow 0$ implies that $d\left(F\left(x_{n}, y_{n}\right), F(x, y)\right) \rightarrow 0$.

Lemma 2.3 (see [6]). Let $(X, d)$ be a CNS. Then $f:(X, d) \rightarrow(X, d)$ is continuous if and only if $f$ is sequentially continuous. 
Definition 2.4 (see $[10,17,18]$ ). Let $(X, \leq)$ be partially ordered set and $F: X \times X \rightarrow X$. F is said to have mixed monotone property if $F(x, y)$ is monotone nondecreasing in $x$ and is monotone nonincreasing in $y$, that is, for any $x, y \in X$,

$$
\begin{aligned}
& x_{1} \preceq x_{2} \Longrightarrow F\left(x_{1}, y\right) \preceq F\left(x_{2}, y\right), \quad \text { for } x_{1}, x_{2} \in X, \\
& y_{1} \preceq y_{2} \Longrightarrow F\left(x, y_{2}\right) \preceq F\left(x, y_{1}\right), \quad \text { for } y_{1}, y_{2} \in X .
\end{aligned}
$$

Note that this definition reduces the notion of mixed monotone function on $\mathbb{R}^{2}$ where $\preceq$ represents usual total order $\leq$ in $\mathbb{R}^{2}$.

Definition 2.5 (see $[10,17,18]$ ). An element $(x, y) \in X \times X$ is said to be a couple fixed point of the mapping $F: X \times X \rightarrow X$ if

$$
F(x, y)=x, \quad F(y, x)=y
$$

Throughout this paper, let $(X, \preceq)$ be partially ordered set and let $d$ be a cone metric on $X$ such that $(X, d)$ is a complete CMS over the normal cone $P$ with the normal constant $K$. Further, the product spaces $X \times X$ satisfy the following:

$$
(u, v) \preceq(x, y) \Longleftrightarrow u \leq x, \quad y \preceq v ; \quad \forall(x, y),(u, v) \in X \times X
$$

Definition 2.6 (see [3]). Let $(X, d)$ be a CMS and $A \subset X$. $A$ is said to be sequentially compact if for any sequence $\left\{x_{n}\right\}$ in $A$ there is a subsequence $\left\{x_{n_{k}}\right\}$ of $\left\{x_{n}\right\}$ such that $\left\{x_{n_{k}}\right\}$ is convergent in $A$.

Remark 2.7 (see [19]). Every cone metric space $(X, d)$ is a topological space which is denoted by $\left(X, \tau_{c}\right)$. Moreover, a subset $A \subset X$ is sequentially compact if and only if $A$ is compact.

Definition 2.8. Let $K$ be a nonempty subset of a CNS $(X, d)$. A set-valued map $H: K \rightarrow 2^{X}$ is called KKM map if for every finite subset $\left\{x_{1}, x_{2}, \ldots, x_{n}\right\}$ of $K$

$$
\operatorname{co}\left\{x_{1}, x_{2}, \ldots, x_{n}\right\} \subset \bigcup_{i=1}^{n} H\left(x_{i}\right)
$$

where co denotes the convex hull.

Lemma 2.9. Let $X$ be a topological vector space, let $K$ be a nonempty subset of $X$, and let $H$ : $K \rightarrow 2^{X}$ be called KKM map with closed values. If $H(x)$ is compact for at least one $x \in K$, then $\bigcap_{x \in K} H(x) \neq \emptyset$. 
Theorem 2.10. Let $\left(X,\|\cdot\|_{P}\right)$ be a CNS over strongly minidhedral cone $P$, and let $K$ be a nonempty convex compact subset of $X$. If $F: K \times K \rightarrow X$ is continuous mapping and $g: K \rightarrow X$ is continuous almost quasiconvex mapping with respect to $F(K \times K)$, then there exists $\left(x_{0}, y_{0}\right) \in K \times K$ such that

$$
\begin{aligned}
\left\|g\left(x_{0}\right)-F(x, y)\right\|_{P}+\left\|g\left(y_{0}\right)-F(y, x)\right\|_{P} \\
\quad=\inf _{(x, y) \in K \times K}\left\{\|g(x)-F(x, y)\|_{P}+\|g(y)-F(y, x)\|_{P}\right\} .
\end{aligned}
$$

Proof. Let $H: K \times K \rightarrow 2^{K \times K}$ by

$$
\begin{gathered}
H(u, v)=\left\{(x, y) \in K \times K:\left\|g(x)-F\left(x_{0}, y_{0}\right)\right\|_{P}+\left\|g(y)-F\left(y_{0}, x_{0}\right)\right\|_{P}\right. \\
\left.\leq\left\|g(u)-F\left(x_{0}, y_{0}\right)\right\|_{P}+\left\|g(v)-F\left(y_{0}, x_{0}\right)\right\|_{P}\right\}
\end{gathered}
$$

for each $(u, v) \in K \times K$. Since $(u, v) \in H(u, v)$, then $H(u, v) \neq \emptyset$. Regarding that the mappings $F$ and $g$ are continuous, $H(u, v)$ is closed for each $(u, v)$. Since $K$ is compact, then $H(u, v)$ is compact for each $(u, v)$. Thus, $H$ is a KKM map.

Let $\left(u_{i}, v_{j}\right) \in K \times K, i \in I, j \in J$ where $I$ and $J$ are finite subsets of $\mathbb{N}$. Then, there exists

$$
\left(u_{0}, v_{0}\right) \in \operatorname{co}\left\{\left(u_{i}, v_{j}\right):(i, j) \in I \times J\right\}, \quad \text { so that }\left(u_{0}, v_{0}\right) \notin \cup\left\{H\left(u_{i}, v_{j}\right):(i, j) \in I \times J\right\} \text {. }
$$

From the first expression in (2.7), one can get that there exist $t_{i j} \geq 0,(i, j) \in I \times J$ such that $\left(u_{0}, v_{0}\right)=\sum_{(i, j) \in I \times J} t_{i j}\left(u_{i}, v_{j}\right)$ and $\sum_{(i, j) \in I \times J} t_{i j}=1$. Set $t_{i}=\sum_{j \in J} t_{i j}$ and $z_{j}=\sum_{i \in I} t_{i j}$ then $\sum_{j \in J} z_{j}=1, \sum_{i \in I} t_{i}=1$ and $\sum_{j \in J} z_{j} v_{j}=v_{0}, \sum_{i \in I} t_{i} u_{i}=u_{0}$. Regarding that $g$ is almost quasiconvex with respect to $F: K \times K \rightarrow X$ yields

$$
\begin{aligned}
& \left\|g\left(u_{0}\right)-F\left(u_{0}, v_{0}\right)\right\|_{P} \leq c_{g}\left(\left(u_{0}\right), F\left(u_{0}, v_{0}\right)\right) \\
& \left\|g\left(v_{0}\right)-F\left(v_{0}, u_{0}\right)\right\|_{P} \leq c_{g}\left(\left(v_{0}\right), F\left(v_{0}, v_{0}\right)\right)
\end{aligned}
$$

where $c_{g}\left(u_{0}, F\left(u_{0}, v_{0}\right)\right) \in\left\{\left\|g\left(u_{i}\right)-F\left(u_{0}, v_{0}\right)\right\|_{P}: i \in I\right\}$ and $c_{g}\left(v_{0}, F\left(v_{0}, u_{0}\right)\right) \in\left\{\| g\left(v_{j}\right)-\right.$ $\left.F\left(v_{0}, u_{0}\right) \|_{P}: j \in J\right\}$.

Thus

$$
\begin{aligned}
& \left\|g\left(u_{0}\right)-F\left(u_{0}, v_{0}\right)\right\|_{P}+\left\|g\left(v_{0}\right)-F\left(v_{0}, u_{0}\right)\right\|_{P} \\
& \quad \leq \inf \left\{\left\|g\left(u_{i}\right)-F\left(u_{0}, v_{0}\right)\right\|_{P}: i \in I\right\}+\inf \left\{\left\|g\left(v_{j}\right)-F\left(v_{0}, u_{0}\right)\right\|_{P}: j \in J\right\} .
\end{aligned}
$$

Taking (2.7) into account, one can get

$$
\left\|g\left(u_{0}\right)-F\left(u_{0}, v_{0}\right)\right\|_{P}+\left\|g\left(v_{0}\right)-F\left(v_{0}, u_{0}\right)\right\|_{P}>\left\|g\left(u_{i}\right)-F\left(u_{0}, v_{0}\right)\right\|_{P}+\left\|g\left(v_{j}\right)-F\left(v_{0}, u_{0}\right)\right\|_{P}
$$


for all $(i, j) \in I \times J$ which is a contradiction. Hence $H$ is a KKM mapping. It follows that there exists $\left(x_{0}, y_{0}\right) \in K \times K$ such that $\left(x_{0}, y_{0}\right) \in H(x, y)$ for all $(x, y) \in K \times K$. Thus,

$$
\begin{aligned}
& \left\|g\left(x_{0}\right)-F\left(x_{0}, y_{0}\right)\right\|_{P}+\left\|g\left(y_{0}\right)-F\left(y_{0}, x_{0}\right)\right\|_{P} \\
& \quad \leq\left\|g(x)-F\left(x_{0}, y_{0}\right)\right\|_{P}+\left\|g(y)-F\left(y_{0}, x_{0}\right)\right\|_{P^{\prime}} \quad \forall(x, y) \in K \times K .
\end{aligned}
$$

Theorem 2.11. Let $\left(X,\|\cdot\|_{P}\right)$ be a CNS over strongly minidhedral cone $P$, and let $K$ be a nonempty convex compact subset of $X$. If $F: K \times K \rightarrow X$ is continuous mapping and $g: X \rightarrow K$ is continuous almost quasiconvex mapping with respect to $F(K \times K)$ such that $F(K \times K) \subset g(K)$, then $F$ and $g$ have a coupled coincidence point.

Proof. Due to Theorem 2.10, there exists $\left(x_{0}, y_{0}\right) \in K \times K$ such that

$$
\begin{aligned}
& \left\|g\left(x_{0}\right)-F\left(x_{0}, y_{0}\right)\right\|+\left\|g\left(y_{0}\right)-F\left(y_{0}, x_{0}\right)\right\|_{P} \\
& \quad=\inf _{(x, y) \in K \times K}\left\{\left\|g(x)-F\left(x_{0}, y_{0}\right)\right\|_{P}+\left\|g(y)-F\left(y_{0}, x_{0}\right)\right\|_{P}\right\} .
\end{aligned}
$$

Since $F(K \times K) \subset g(K)$,

$$
\inf _{(x, y) \in K \times K}\left\{\left\|g(x)-F\left(x_{0}, y_{0}\right)\right\|_{P}+\left\|g(y)-F\left(y_{0}, x_{0}\right)\right\|_{P}\right\}=0,
$$

then $\left\|g\left(x_{0}\right)-F\left(x_{0}, y_{0}\right)\right\|_{P}+\left\|g\left(y_{0}\right)-F\left(y_{0}, x_{0}\right)\right\|_{P}=0$.

Thus, $g\left(x_{0}\right)=F\left(x_{0}, y_{0}\right)$ and $g\left(y_{0}\right)=F\left(y_{0}, x_{0}\right)$.

If we take $g: K \rightarrow X$ as an identity, $g(x)=x$, in Theorem 2.11, then we get the following result.

Theorem 2.12. Let $\left(X,\|\cdot\|_{P}\right)$ be a CNS over strongly minidhedral cone $P$, and let $K$ be a nonempty convex compact subset of $X$. If $F: K \times K \rightarrow K$ is continuous mapping, then $F$ has a coupled fixed point.

Theorem 2.13. Let $\left(X,\|\cdot\|_{P}\right)$ be a CNS over strongly minidhedral cone $P$, and let $K$ be a nonempty convex compact subset of $X$. If $F: K \times K \rightarrow X$ is continuous mapping, then either $F$ has a coupled fixed point or there exists $\left(x_{0}, y_{0}\right) \in(\partial K \times K \cup K \times \partial K)$ such that

$$
0<\left\|x_{0}-F\left(x_{0}, y_{0}\right)\right\|_{P}+\left\|y_{0}-F\left(y_{0}, x_{0}\right)\right\|_{P} \leq\left\|x-F\left(x_{0}, y_{0}\right)\right\|_{P}+\left\|y-F\left(y_{0}, x_{0}\right)\right\|_{P}
$$

for all $(x, y) \in K \times K$. 
Proof. If $F$ has a coupled fixed point, then we are done. Suppose that $F$ has no coupled fixed points. Due to Theorem 2.10, there exists $\left(x_{0}, y_{0}\right) \in K \times K$ such that

$$
\begin{aligned}
& \left\|g\left(x_{0}\right)-F\left(x_{0}, y_{0}\right)\right\|_{P}+\left\|g\left(y_{0}\right)-F\left(y_{0}, x_{0}\right)\right\|_{P} \\
& \quad=\inf _{(x, y) \in K \times K}\left\{\left\|g(x)-F\left(x_{0}, y_{0}\right)\right\|_{P}+\left\|g(y)-F\left(y_{0}, x_{0}\right)\right\|_{P}\right\} .
\end{aligned}
$$

Take $g(x)=x$ which implies (2.14). It is sufficient to show that $\left(x_{0}, y_{0}\right) \in(\partial K \times K \cup K \times \partial K)$. The inequality (2.14) implies that either $F\left(x_{0}, y_{0}\right) \notin K$ or $F\left(y_{0}, x_{0}\right) \notin K$.

Consider the first case: $F\left(x_{0}, y_{0}\right) \notin K$. Suppose $x_{0} \in \operatorname{int}(K)$. Since $K$ is convex, then there exists $t \in(0,1)$ such that $x=t x_{0}+(1-t) F\left(x_{0}, y_{0}\right) \in K$. Thus $\left\|x-F\left(x_{0}, y_{0}\right)\right\|_{P}=t \| x_{0}-$ $F\left(x_{0}, y_{0}\right) \|_{P}$ and

$$
\inf _{x \in K}\left\|x-F\left(x_{0}, y_{0}\right)\right\|_{P} \leq t\left\|x_{0}-F\left(x_{0}, y_{0}\right)\right\|_{P}<\left\|x_{0}-F\left(x_{0}, y_{0}\right)\right\|_{P}
$$

This is a contradiction. Analogously one can get the contradiction from the case $F\left(y_{0}, x_{0}\right) \notin K$. Thus, $\left(x_{0}, y_{0}\right) \in(\partial K \times K \cup K \times \partial K)$.

Theorem 2.14. Let $\left(X,\|\cdot\|_{P}\right)$ be a CNS over strongly minidhedral cone $P$, and let $K$ be a nonempty convex compact subset of $X$. Suppose that $F: K \times K \rightarrow X$ is continuous mapping. Then $F$ has a coupled fixed point if one of the following conditions is satisfied for all $\left(x_{0}, y_{0}\right) \in(\partial K \times K \cup K \times \partial K)$ such that $(x, y) \neq(F(x, y), F(y, x))$ :

(i) there exists $a(u, v) \in K \times K$ such that

$$
\|u-F(x, y)\|_{P}<\|x-F(x, y)\|_{P^{\prime}} \quad\|v-F(y, x)\|_{P}<\|y-F(y, x)\|_{P^{\prime}}
$$

(ii) there exists an $t \in(0,1)$ such that

$$
K \cap\left(B\left(F(x, y), t\|x-F(x, y)\|_{P}\right)\right) \neq \emptyset, \quad K \cap\left(B\left(F(y, x), t\|y-F(y, x)\|_{P}\right)\right) \neq \emptyset
$$

(iii) $\{F(x, y), F(y, x)\} \subset K$.

Proof. It is clear that (iii) $\Rightarrow$ (ii) $\Rightarrow$ (i). To finalize proof, it is sufficient to show that $(i)$ is satisfied. Suppose that $(i)$ holds but $F$ has no coupled fixed point. Take Theorem 2.13 into account; then there exist $\left(x_{0}, y_{0}\right) \in(\partial K \times K \cup K \times \partial K)$ such that (2.14) holds which contradicts $(i)$. 


\section{References}

[1] B. Rzepecki, "On fixed point theorems of Maia type," Publications de l'Institut Mathématique, vol. 28, pp. 179-186, 1980.

[2] S.-D. Lin, "A common fixed point theorem in abstract spaces," Indian Journal of Pure and Applied Mathematics, vol. 18, no. 8, pp. 685-690, 1987.

[3] L.-G. Huang and X. Zhang, "Cone metric spaces and fixed point theorems of contractive mappings," Journal of Mathematical Analysis and Applications, vol. 332, no. 2, pp. 1468-1476, 2007.

[4] M. S. Khan and M. A. Imdad, "A common fixed point theorem for a class of mappings," Indian Journal of Pure and Applied Mathematics, vol. 14, no. 10, pp. 1220-1227, 1983.

[5] Sh. Rezapour and R. Hamlbarani, "Some notes on the paper: "cone metric spaces and fixed point theorems of contractive mappings"," Journal of Mathematical Analysis and Applications, vol. 345, no. 2, pp. 719-724, 2008.

[6] D. Turkoglu and M. Abuloha, "Cone metric spaces and fixed point theorems in diametrically contractive mappings," Acta Mathematica Sinica, vol. 26, no. 3, pp. 489-496, 2010.

[7] D. Turkoglu, M. Abuloha, and T. Abdeljawad, "KKM mappings in cone metric spaces and some fixed point theorems," Nonlinear Analysis: Theory, Methods \& Applications, vol. 72, no. 1, pp. 348-353, 2010.

[8] C. Di Bari and P. Vetro, " $\phi$-pairs and common fixed points in cone metric spaces," Rendiconti del Circolo Matematico di Palermo, vol. 57, no. 2, pp. 279-285, 2008.

[9] E. Karapinar, "Fixed point theorems in cone Banach spaces," Fixed Point Theory and Applications, Article ID 609281, 9 pages, 2009.

[10] E. Karapınar, "Couple fixed point theorems for nonlinear contractions in cone metric spaces," Computers and Mathematics with Applications, vol. 59, no. 12, pp. 3656-3668, 2010.

[11] S. Radenović and B. E. Rhoades, "Fixed point theorem for two non-self mappings in cone metric spaces," Computers \& Mathematics with Applications, vol. 57, no. 10, pp. 1701-1707, 2009.

[12] Z. D. Mitrović, "A coupled best approximations theorem in normed spaces," Nonlinear Analysis: Theory, Methods \& Applications, vol. 72, no. 11, pp. 4049-4052, 2010.

[13] T. Abdeljawad and E. Karapınar, "Quasicone metric spaces and generalizations of Caristi Kirk's theorem," Fixed Point Theory and Applications, Article ID 574387, 9 pages, 2009.

[14] K. Deimling, Nonlinear Functional Analysis, Springer, Berlin, Germany, 1985.

[15] T. Abdeljawad, "Completion of cone metric spaces," Hacettepe Journal of Mathematics and Statistics, vol. 39, no. 1, pp. 67-74, 2010.

[16] T. Abdeljawad, D. Turkoglu, and M. Abuloha, "Some theorems and examples of cone Banach spaces," Journal of Computational Analysis and Applications, vol. 12, no. 4, pp. 739-753, 2010.

[17] T. Gnana Bhaskar and V. Lakshmikantham, "Fixed point theorems in partially ordered metric spaces and applications," Nonlinear Analysis: Theory, Methods \& Applications, vol. 65, no. 7, pp. 1379-1393, 2006.

[18] V. Lakshmikantham and L. Cirić, "Coupled fixed point theorems for nonlinear contractions in partially ordered metric spaces," Nonlinear Analysis: Theory, Methods \& Applications, vol. 70, no. 12, pp. 4341-4349, 2009.

[19] Sh. Rezapour, M. Derafshpour, and R. Hamlbarani, “A review on topological properties of cone metric spaces," to appear. 